\title{
Stem cells for multiple sclerosis: promises and reality
}

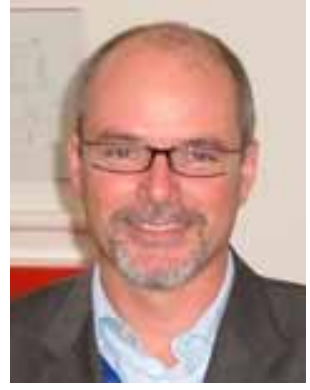

\section{AntanioUcadlit, Francesco Frastoni ${ }^{2} \&$ Gianluig Mancardi ${ }^{1}$}

${ }^{\dagger}$ Author for correspondence ${ }^{1} \mathrm{~N}$ euroimmunology U nit, D epartment of N eurosciences, $O$ phthalmology and $G$ enetics, University of Genoa, Via D e Toni 5, 16132, Genoa, Italy Tel.: +39010353 7028; Fax: +390103538639;

E-mail: auccelli@ neurologia.unige.it ${ }^{2}$ Centre for Stem Cell and Cell Therapy, D epartment of $\mathrm{H}$ aematology, San M artino H ospital, 16132, Genoa, Italy

Tel.: +39010 555 3943; E-mail: francesco.frassoni@ hsanmartino.liguria.it

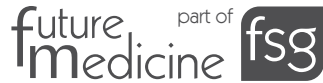

'Stem cell thera py for the trea tment of MS is c urrently seen asan a p pealing tool to replace damaged cells in MS, enhance remyelination, regenera te the deranged neural network and restore nerve functions.'

Stem cells are heterogeneous cell populations, which are often mistakenly considered capable of repairing almost every tissue because of their intrinsic capacity of differentiating in cells of every tissue lineage. Based on these expectations, stem cells have been proposed for the treatment of diseases where tissue degeneration occurs, often as consequence of inflammation, as in multiple sclerosis (M S).

In M S, a dysregulated immune response, possibly trigged by environmental factors such as a microbial trigger, occurs in genetically susceptible individuals. In this scenario, autoimmunityprone $T$ cells are activated in the periphery by a pathogen, possibly due to an inefficient control by regulatory systems, such as regulatory $T$ cells, and, as a consequence, upregulate adhesion molecules necessary to cross the brain endothelium. Upon migration into the CNS, autoreactive $T$ cells are further activated by local antigen-presenting cells (APCS), such as microglia, and initiate a complex attack against myelin antigens through the recruitment of several other immune cells, including B cells, overall leading to myelin disintegration and impairment of nerve conduction.

An uncontrolled autoimmune response is likely to sustain recruitment of other pathogenic cells from the periphery leading, at later stages, to the compartmentalization of the pathogenic attack inside the CNS. Chronic aggression against the myelin sheath will eventually lead to axonal sufferance and subsequent loss of neurons and oligodendrocytes, the biological basis of irreversible disability. The ideal treatment for MS should therefore target the autoimmune attack and support tissue repair or at least tissue protection. Indeed, tissue repair without the arrest of the cause of tissue damage would be ineffective.
Stem cell therapy for the treatment of MS is currently seen as an appealing tool to replace damaged cells in MS, enhance remyelination, regenerate the deranged neural network and restore nerve functions. However, in our opinion, at least four major points have to be addressed in order to efficiently exploit stem cells for the treatment of M S.

First, current data show that adult stem cells may differentiate into neural cells under some experimental conditions. In spite of this, adult stem cells, independently of their source, havea limited capacity to differentiate into neural cells upon in vivo transplantation and are unlikely to be able to recre ate the complex neural network required for the recapitulation of lost functions. To this end, stem cell approaches must address the issue of enhancing terminal differentiation into cells of the injured tissue, such as neural cellsin M S.

Second, multifocal lesions involving different areas spread all over the CN S characterize MS. Therefore, therapeutic stem cells would need to reach all the injured areas of the CNS in order to effectively contribute to tissue healing. $0 \mathrm{n}$ this, the route of administration should allow stem cells to home in the CN S and diffuse into the damaged tissue liberally.

Third, any effective cell therapy approach aimed at tissue repair and protection should be combined with an intervention, such as immunosuppressive agents, aimed at blocking the autoimmune response that otherwise would rapidly target the transplanted cells. This aspect would be particularly important in case of an allogeneic source for stem cells.

Fourth, due to the chronic nature of $\mathrm{M} \mathrm{S}$, a disease characterized by a prominent inflammation occurring in its early phase, followed by a subsequent loss of myelin and neurons often taking place many years after disease onset, efforts should be taken to identify time windows along disease course where the administration of stem cells could have a reasonable effect before the occurrence of irreparable disability. At present, there is no experimental evidence that administration of adult stem cells at stages where neural tissues have been irreversibly injured could lead to the reconstruction of the damaged organ. 
Recent studies conducted in experimental autoimmune encephalomyelitis (EAE), the animal model for MS, have demonstrated that at least two populations of adult stem cells could be promising tools for the treatment of M S.

Bone marrow-derived stroma contains a subset of mesenchymal progenitor cells named mesenchymal stem cells (M SC s) capable of supporting hematopoiesis and differentiating into cell types of the mesodermal lineage but also, in some experimental conditions, into other cells types, including neural cells [1]. M SC s have been used already in humans to foster engraftment of hematopoietic stem cells (HSCs) upon allogeneic bone marrow transplantation [2] and for the treatment of osteogenesis imperfecta [3]. Recently, M SC s have been shown to affect many effector functions of the cells of adaptive immunity, particularly when exerted under inflammatory/Th1-oriented conditions [4]. In particular, MSC S inhibit proliferation of activated $T$ and $B$ cells inducing the arrest of the cell cycle into the $G_{0}$ phase. These findings provided the rationale to propose M SC s for the treatment of acute graftversus-host disease (GVHD), a disease characterized by a pronounced attack of the grafted immune cells against the recipient of an allogeneic incompatible bone marrow. In this human disease, MSCS have been shown to reverse treatment-refractory G VH D [5].

Based on the capacity of M SC s to inhibit Tand B-cell effector function and their supposed ability to transdifferentiate into neural cells, we sought to utilize it for the treatment of EAE. We have shown that intravenous injection of M SC S in EAE-affected mice leads to a striking amelioration of disease clinical course and reduces inflammation and demyelination [6]. This beneficial effect was obtained when mice were treated early after disease onset and was associated with an impairment of the Tand $\mathrm{B}$-cell response against myelin antigens detected at the level of the lymphoid organs, suggesting the induction of peripheral tolerance. By contrast, M SC S reached the CNS only after disease chronicization, migrating close to the inflammatory infiltrates but without significantly differentiating into neural cells. However, we observed a decreased amount of axonal loss associated with an increased number of neurons in the inflamed areas of the CN S, suggesting that M SC S could foster survival of dying neurons [7]. These results are in line with the gene expression profile of therapeutic M SC s, which is characterized by the abundant presence of molecules known to have an anti-apoptotic effect together with an immunomodulatory activity (PedemonteE et al., Unpublished D ata).

In summary, these results suggest that M SCS could be administered intravenously in the early phase of MS, aiming to block the autoimmune response in the periphery and protect neural cells from death through the release of anti-apoptotic factors.

Another population of adult stem cells, namely neural progenitor cells (NPCs) has been demonstrated to be able to ameliorate EAE upon intravenous or intracisternal injection at different stages of the disease [8]. U pon intravenous administration, NPCS migrated into the CNS by means of the same adhesion molecules and chemokine receptors used by $T$ cells to interact with the inflamed endothelium. A limited number of the transplanted NPCs differentiated into neural cells and released growth factors, leading to the recruitment of local precursor cells and subsequent attempt to repair the damaged tissue. In addition, a fraction of transplanted N PC s remained in an undifferentiated $\left(\mathrm{Nestin}^{+}\right)$state within perivascular areas where, closely interacting with immune and neural cells, could counteract the deleterious effect of encephalitogenic T cells [9]. Interestingly, it has been shown that transplanted N PCs migrate specifically to the injured CNS under the guidance of immune cells that may aid in directing stem cells towards local disease sites [10]. In addition, similarly to that observed for M SC s, N PC s appear to be able to reach lymphoid organs and induce peripheral immune tolerance [11].

These studies support the possible translation of these results into clinical attempts to treat M S. H owever, irrespective of what may be expected by most people in terms of tissue repair, current evidence indicate that MSCs and NPCs will not be able to regenerate irreversibly damaged tissues and restore nerve conduction and lost functions. Based on their immunomodulatory properties, their ability to rel ease growth factors and anti-apoptotic molecules and their capacity of migrating at the site of inflammation inside the CN S upon intravenous administration, it would be reasonable to exploit stem cells for the treatment of severe, rapidly evolving cases of MS where a high inflammatory load is present and inexorably moves toward tissue destruction. 


\section{Stem cells for multiple sclerosis: promises and reality - EDIT O RIAL}

Despite these encouraging results, several safety concerns must be resolved before moving securely into clinical studies. While the safety profile of N PCs is unknown due to the lack of clinical studies, MSCs have been al ready utilized in several life-threatening conditions, including GVHD, with a relatively safe peri-transplant profile. H owever, the long and chronic disease course of MS, together with the vanishing inflammation occurring during the late phases of disease where tissue damage occurs, hinders their unrestricted use in such disease and requires an accurate evaluation of their safety profile with specific attention to their potential induction of and/or differentiation into tumor cells [12]. In addition, the conflicting data regarding their ability to escape an immune response upon allogeneic transplantation [13] raises concerns about their source (allogeneic vs autologous) and the opportunity of a therapeutic association with some immunosuppressive agent.

Thus, while preclinical data have already provided a strong rationale for the utilization of stem cellsfor M S, other studies will have to challenge their capacity to differentiate into neural cells and stimulate en dogenous neurogenesis and to address their safety profile in often very disabling but mostly not life threatening diseases, such as MS.
Bibliography

1. Pittenger M F, M ackay AM, Beck SC et al.: M ultilineage potential of adult human mesenchymal stem cells. Science 284(5411), 143-147 (1999).

2. Lazarus $H M, K$ Ko $O N$, D evine SM et al.: Cotransplantation of H LA-identical sibling culture expanded mesenchymal stem cells and hematopoietic stem cells in hematologic malignancy patients. Biol. Blood M arrow Transplant. 11(5), 389-398 (2005).

3. H orwitz EM, Gordon PL, Koo WKK et al.: Isolated allogeneic bone marrow-derived mesenchymal cells engraft and stimulate growth in children with osteogenesis imperfecta: implications for cell therapy of bone. Proc. Natl Acad. Sci. USA 99(13), 8932-8937 (2002).

4. U ccelli A, M oretta L, Pistoia V. Immunoregulatory function of mesenchymal stem cells. Eur. J. Immunol. 36(60), 2566-2573 (2006).
5. Le Blanc K, Rasmusson I, Sundberg B et al. Treatment of severe acute graft-versus-host disease with third party haploidentical mesenchymal stem cells. Lancet. 363(9419), 1439-1441 (2004).

6. Zappia E, Casazza S, Pedemonte E et al.: $M$ esenchymal stem cells ameliorate experimental autoimmune encephalomyelitis inducing $T$ cell anergy. Blood 106(5), 1755-1761 (2005).

7. Gerdoni $E$, Gallo $B, C$ asazza $S$ et al.: $M$ esenchymal stem cells effectively modulate pathogenic immune response in EAE. Ann. N erol. (2007) (In Press).

8. Pluchino $S, Q$ uattrini $A$, Brambilla $E$ et al.: Injection of adult neurospheres induces recovery in a chronic model of multiple sclerosis. N ature 422(6933), 688-694 (2003).

9. Pluchino $S$, Zanotti $L$, Rossi B et al.: N eurosphere-derived multipotent precursors promote neuroprotection by an immunomodulatory mechanism. $\mathrm{N}$ ature 436(7048), 266-271 (2005).
10. Ben $\mathrm{H}$ ur T, Einstein O, M izrachi-Kol R et al.: Transplanted multipotential neural precursor cells migrate into the inflamed white matter in response to experimental autoimmune encephalomyelitis. Glia 41(1), 73-80 (2003).

11. Einstein 0 , Fainstein $\mathrm{N}$, Vaknin I et al.: $N$ eural precursors attenuate autoimmune encephalomyelitis by peripheral immunosuppression. Ann. N eurol. (2007) (In Press).

12. Tolar J, N auta AJ, $\mathrm{O}$ sborn $\mathrm{MJ}$ et al. Sarcoma derived from cultured mesenchymal stem cells. Stem Cells. (2006) [Epub ahead of print].

13. N auta AJ, Westerhuis $G$, Kruisselbrink $A B$ et al.: D onor-derived mesenchymal stem cells are immunogenic in an allogeneic host and stimulate donor graft rejection in a nonmyeloablative setting. Blood 108(6), 2114-2120 (2006). 